\title{
Optimal Fat Suppression in Head and Neck MRI: Comparison of Multipoint Dixon with 2 Different Fat-Suppression Techniques, Spectral Presaturation and Inversion Recovery, and STIR
}

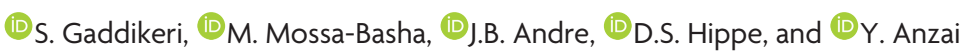

\begin{abstract}
BACKGROUND AND PURPOSE: Uniform complete fat suppression is essential for identification and characterization of most head and pathology. Our aim was to compare the multipoint Dixon turbo spin-echo fat-suppression technique with 2 different fat-suppression techniques, including a hybrid spectral presaturation with inversion recovery technique and an inversion recovery STIR technique, in head and neck fat-suppression MR imaging.
\end{abstract}

MATERIALS AND METHODS: Head and neck MR imaging datasets of 72 consecutive patients were retrospectively reviewed. All patients were divided into 2 groups based on the type of fat-suppression techniques used (group A: STIR and spectral presaturation with inversion recovery gadolinium-TIWI; group B: multipoint Dixon T2 TSE and multipoint Dixon gadolinium-TIWI TSE). Objective and subjective image quality and scan acquisition times were assessed and compared between multipoint Dixon T2 TSE versus STIR and multipoint Dixon gadolinium-TIWI TSE versus spectral presaturation with inversion recovery gadolinium-TIWI using the Mann-Whitney $U$ test.

RESULTS: A total of 64 patients were enrolled in the study (group A, $n=33$ and group B, $n=31$ ). Signal intensity ratios were significantly higher for multipoint Dixon T2 and gadolinium-TIWI techniques compared with STIR $(P<.001)$ and spectral presaturation with inversion recovery gadolinium-TIWI $(P<.001)$, respectively. Two independent blinded readers revealed that multipoint Dixon T2 and gadoliniumTTWI techniques had significantly higher overall image quality $(P=.022$ and $P<.001)$ and fat-suppression grades $(P<.013$ and $P<.001$ across 3 different regions) than STIR and spectral presaturation with inversion recovery gadolinium-TIWI, respectively. The scan acquisition time was relatively short for the multipoint Dixon technique ( 2 minutes versus 4 minutes 56 seconds for the T2-weighted sequence and 2 minutes versus 3 minutes for the gadolinium-TIWI sequence).

CONCLUSIONS: The multipoint Dixon technique offers better image quality and uniform fat suppression at a shorter scan time compared with STIR and spectral presaturation with inversion recovery gadolinium-TIWI techniques.

ABBREVIATIONS: AP = anteroposterior; CHESS = chemical shift selective suppression; FS = fat suppression; Gad-TIWI = gadolinium-TIWI; $\mathrm{mDixon}=$ multipoint Dixon; SPIR = spectral presaturation with inversion recovery

U niform and complete fat suppression (FS) is indispensable for accurate diagnosis and characterization of head and neck pathologies. Various FS MR imaging techniques are avail-

\footnotetext{
Received March 3, 2017; accepted after revision October 16.

From the Departments of Neuroradiology (S.G.) and Radiology (S.G.), Rush University Medical Center, Chicago, Illinois; Departments of Neuroradiology (M.M.-B.) and Radiology (D.S.H.), University of Washington, Seattle, Washington; Department of Neuroradiology (J.B.A.), University of Washington Medical Center, Seattle, Washington; and Department of Radiology (Y.A.), University of Utah Health Center, Salt Lake City, Utah.

Paper previously presented at: Annual Meeting of the American Society of Neuroradiology and the Foundation of the ASNR Symposium, April 22-27, 2017; Long Beach, California.

Please address correspondence Santhosh Gaddikeri, MD, Department of Radiology, Rush University Medical Center, 1653 W Congress Parkway, Chicago, IL 60612; e-mail: Santhosh_Gaddikeri@rush.edu

三 Indicates article with supplemental on-line table.

http://dx.doi.org/10.3174/ajnr.A5483
}

able clinically, each with its own advantages and disadvantages. Commonly used FS MR imaging techniques include STIR, chemical shift selective suppression (CHESS), hybrid methods such as spectral presaturation with inversion recovery (SPIR; Phillips Healthcare, Best, the Netherlands), spectral attenuated inversion recovery, and a more recent chemical shift method, the multipoint Dixon (mDixon Technique; Phillips Healthcare).

The STIR technique nulls the fat signal using a $180^{\circ}$ inversion pulse as an initial excitation pulse, followed by a subsequent $90^{\circ}$ pulse at a specified inversion time (approximately $160-180 \mathrm{~ms}$ for a $1.5 \mathrm{~T}$ magnet). The CHESS technique uses a radiofrequency pulse tuned to the fat-resonance frequency together with a spoiler gradient, which saturates fat signal and thus leaves only water protons to produce signal. SPIR is a hybrid FS technique that combines the fat selectivity of CHESS and uses an inversion radiofrequency pulse like that in the STIR technique. Never- 
Table 1: Parameters used for different fat-suppression sequences on a 3T scanner ${ }^{\mathrm{a}}$

\begin{tabular}{|c|c|c|c|c|}
\hline & Axial STIR & Axial T2WI & Axial Gad-TIWI & Axial Gad-TIWI \\
\hline & TSE & mDixon TSE & SPIR TSE & mDixon TSE \\
\hline \multirow[t]{2}{*}{ Coil } & 16 Channel & 16 Channel & 16 Channel & 16 Channel \\
\hline & SENSE NV & SENSE NV & SENSE NV & SENSE NV \\
\hline $\mathrm{TR} / \mathrm{TE}$ & $3000 / 15 \mathrm{~ms}$ & $3000 / 80 \mathrm{~ms}$ & $600 / 9.2 \mathrm{~ms}$ & $500 / 10 \mathrm{~ms}$ \\
\hline $\begin{array}{l}\text { Section thickness/ } \\
\text { intersection gap }\end{array}$ & $3 / 1 \mathrm{~mm}$ & $3 / 1 \mathrm{~mm}$ & $3 / 1 \mathrm{~mm}$ & $3 / 1 \mathrm{~mm}$ \\
\hline No. of axial images & 40 & 40 & 40 & 40 \\
\hline FS technique & $\begin{array}{l}\text { Inversion recovery } \\
\quad(\mathrm{TI}=200 \mathrm{~ms})\end{array}$ & mDixon & SPIR & mDixon \\
\hline Acquisition matrix & $200 \times 141$ & $232 \times 232$ & $288 \times 196$ & $204 \times 199$ \\
\hline NEX & 2 & 1 & 1 & 1 \\
\hline Acquisition time & $4 \mathrm{~min}, 56 \mathrm{sec}$ & $2 \mathrm{~min}, 2 \mathrm{sec}$ & $3 \mathrm{~min}, 2 \mathrm{sec}$ & $2 \mathrm{~min}, 8 \mathrm{sec}$ \\
\hline Parallel imaging & Yes & Yes & Yes & Yes \\
\hline Gadolinium contrast & N/A & N/A & $\begin{array}{l}0.1 \mathrm{mmol} / \mathrm{kg} \text { gadodiamide } \\
\text { (Gd-DTPA) (ProHance) }\end{array}$ & $\begin{array}{c}0.1 \mathrm{mmol} / \mathrm{kg} \text { gadodiamide } \\
\text { (Gd-DTPA) (ProHance) }\end{array}$ \\
\hline
\end{tabular}

Note:-SENSE indicates sensitivity encoding; N/A, not applicable; NV, NeuroVascular.

${ }^{a}$ Achieva; Philips Healthcare.

theless, SPIR differs from CHESS in that the radiofrequency pulse used is an inversion pulse and is different from STIR in that the inversion pulse used is selective for fat spin excitation only. ${ }^{1}$ The more recently developed mDixon technique is insensitive to magnetic field (both B0 and B1) inhomogeneity while preserving the desired image contrast at reduced scan acquisition times. $^{2}$

In this retrospective study, we compared 3-point mDixon T2 TSE and mDixon gadolinium-T1WI (Gad-T1WI) TSE techniques with the commonly used T2 (STIR) and post-Gad-T1WI (SPIR) FS techniques, respectively, for assessing subjective and objective image quality while considering image-acquisition times.

\section{MATERIALS AND METHODS}

The ethics committee of our institution (University of Washington) approved this Health Insurance Portability and Accountability Act-compliant study. We retrospectively reviewed the imaging records of 72 consecutive patients who had undergone head and neck MR imaging without and with gadolinium administration for various clinical indications, between July 1, 2014, and September 30, 2014. Another inclusion criterion was that these patients also have a CT examination of the neck within 3 months before or after the MR imaging study. The CT data were used to quantify body habitus. Patients with poor renal function (glomerular filtration rate of $<30$ ) and suboptimal image quality secondary to patient motion artifacts were excluded from the study. None of the enrolled patients had cervical spine fusion hardware. Systematic changes were made in the institutional head and neck MR imaging protocol during the study time period so that the previously obtained STIR/SPIR combination of FS techniques was gradually switched to the evaluated mDixon FS techniques. The enrolled subjects were divided into 2 groups (group A and group B) based on the combination of FS techniques used for the corresponding T2-weighted sequence and Gad-T1WI sequence. In group A subjects, STIR images were acquired as a T2-weighted sequence and SPIR images were acquired as the post Gad-T1WI FS technique. In group B subjects, a 2D mDixon spin-echo (2D-3-point mDixon TSE) technique was used to suppress fat signal in both T2-weighted and post-GadT1WI sequences.

\section{Data Acquisition}

All MR imaging scans were obtained on a 3T scanner (Achieva; Phillips Healthcare). As per our institution protocol, we obtained the following MR imaging sequences: axial, sagittal, and coronal T1WI; and axial and coronal fluid-sensitive sequences with fat suppression (axial STIR and coronal CHESS FS T2WI or axial and coronal mDixon FS T2WI), followed by gadolinium-enhanced (gadoteridol, ProHance, 279.3 mg/mL; Bracco Diagnostics, Princeton, New Jersey) axial and coronal T1WI with FS sequences (SPIR or mDixon). Parameters used for STIR, SPIR FS Gad-T1WI, mDixon FS T2WI, and mDixon FS Gad-T1WI are summarized in Table 1.

\section{Data Analysis}

Objective Analysis. All objective measurements were performed by a fellowship-trained neuroradiologist with 10 years of cumulative experience in head and neck imaging. As the surrogate measure of a patient's body size, the maximum anteroposterior (AP) neck diameter at the level of mandible (parallel to the C2-3 intervertebral disc), minimum AP diameter at the midneck level (parallel to the $\mathrm{C} 4-5$ intervertebral disc), and maximum transverse shoulder width (Fig 1) were measured on the neck CT topogram. The ratios of AP neck diameter at the level of the mandible to the AP diameter at the midneck level and shoulder width to AP diameter of the midneck were calculated for each subject and were compared between the 2 groups (groups A and B).

Signal intensity of the spinal cord and subcutaneous fat was measured (Fig 2) by placing a circular ROI measuring 5-10 $\mathrm{mm}$ in diameter on an axial image at 2 different levels (submandibular region and supraclavicular region) on all sequences. To normalize the relative fat signal intensity, we calculated the signal intensity ratio between the spinal cord and subcutaneous fat at both levels for each sequence. The ratios were compared between STIR and mDixon FS T2 TSE and between SPIR FS Gad-T1WI and mDixon FS Gad-T1WI TSE, respectively.

Subjective Analysis. Two fellowship-trained and board-certified neuroradiologists, each with 12 years of experience in in- 

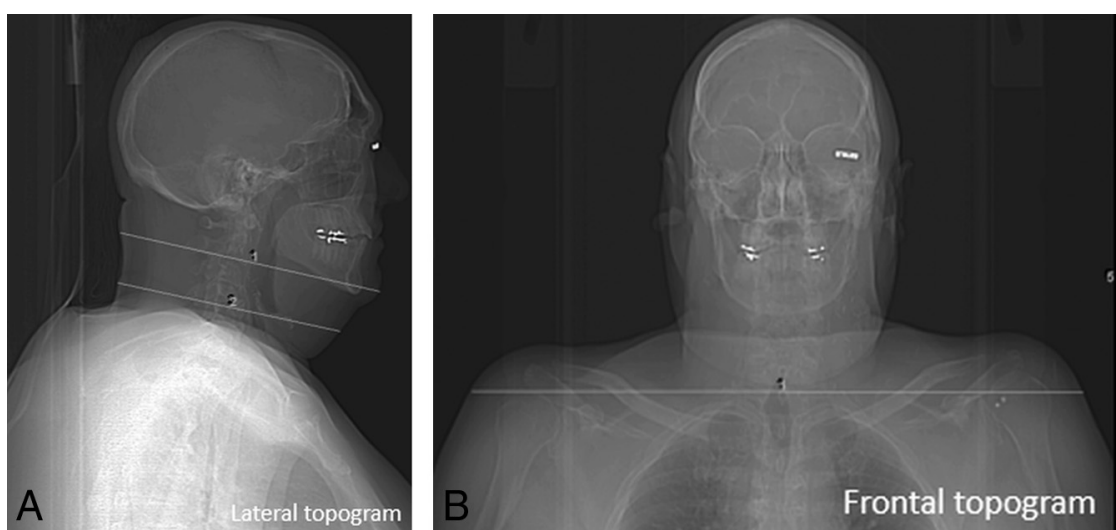

FIG 1. Lateral $(A)$ and frontal $(B)$ projections of $C T$ topogram images with measurements of anteroposterior diameter at the level of $\mathrm{C} 2-3$ and $\mathrm{C} 4-5$ and transverse diameters at the shoulder.
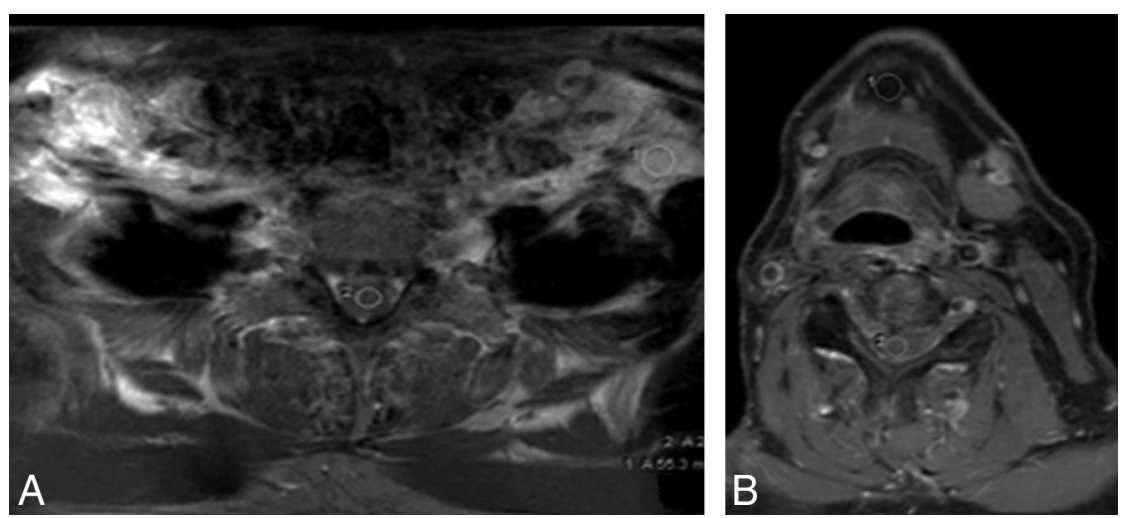

FIG 2. Gadolinium-enhanced T7-weighted MR images with SPIR $(A)$ and mDixon $(B)$ techniques for fat suppression. ROIs are placed on the spinal cord and fat to obtain a signal intensity ratio.

terpreting head and neck MR imaging, independently assessed the image quality. Readers were blinded to all FS techniques used. On a per-subject basis, the stack of axial images of each sequence (FS T2 sequence and FS Gad-T1WI sequence) was displayed and analyzed on a random basis on a PACS monitor. No preset window width or level was provided. The readers were free to vary both at their discretion. The 2 readers were asked to evaluate the following: 1) uniformity of fat suppression with emphasis on 3 areas that are prone to incomplete fat suppression (the maxillary, submandibular, and supraclavicular regions, respectively), 2) overall image quality for each fat-suppression technique, and 3) the presence of susceptibility artifacts from dental amalgam. Uniformity of fat suppression and overall image quality assessment were graded on a 5 -point Likert-like scale ( $1=$ poor, 2 = suboptimal, $3=$ acceptable, $4=$ good, and $5=$ excellent $)$.

Direct comparison of objective and subjective image-quality measurements between groups A and B (STIR versus mDixon T2 TSE and Gad-T1WI SPIR versus mDixon Gad-T1WI TSE) were performed. Sequence-specific acquisition times were collected and compared between the 2 groups.

\section{Statistical Analysis}

Variables were summarized as mean \pm SD or count (percentage). The sequence groups were compared using the Mann-Whitney $U$ test. For the analysis of subjective image-quality ratings, the ratings of 2 readers were averaged to compare the groups. A permutation test, clustered by patient, was used to compare the presence of dental amalgam susceptibility artifacts as rated by both readers separately between sequence groups. Interreader agreement of the subjective ratings was summarized using the Cohen $\kappa$ (linearly weighted for 5-point scales and unweighted for binary variables) and percentage agreement. Percentage agreement for the 5-point scales was calculated after combining the ratings into a 3-point scale: $1-2,3$, and 4-5. Bias in ratings between the readers was assessed using the Wilcoxon signed rank test. A $P$ value of $<.05$ was considered statistically significant. All statistical calculations were conducted with the statistical computing language R (Version 3.1.1; http://www.r-project.org/).

\section{RESULTS \\ Patient Demographics}

Group-based patient demographics are summarized in Table 2. A total of 64 patients met the inclusion criteria, following exclusion of 8 patients (due to suboptimal MR imaging due to motion artifacts $[n=$ 5] and lack of intravenous gadoliniumbased contrast agent administration $[n=$ 3]). Group A comprised 33 patients (10 women), while group B comprised 31 patients (11 women).

There was no statistically significant difference in the age (mean, $61 \pm 15$ years versus $55 \pm 17$ years; $P=.15)$ or $\operatorname{sex}(P=.79)$ distribution, between the groups. There was no significant difference in body habitus, represented by the ratio between groups, of shoulder width-to-mandibular region AP neck diameter at the C2-3 level $(1.53 \pm 0.16$ versus $1.48 \pm 0.15$ for groups $A$ and $B$, respectively; $P=$ .35); and the ratio of shoulder width-to-midneck AP diameter at the C4-5 level (3.21 \pm 0.36 versus $3.16 \pm 0.40$, for groups A and B, respectively; $P=.88)$.

\section{Objective Assessment}

Objective image-quality measurements are summarized in Table 3. Signal intensity ratios measured between the spinal cord and subcutaneous fat at the submandibular and supraclavicular levels were significantly higher for the mDixon technique. For T2-weighted sequences (STIR versus mDixon T2-weighted TSE), the ratios measured $3.5 \pm 3.4$ versus $5.7 \pm 1.6$, respectively $(P<.001)$ at the submandibular level and $3.3 \pm 3.4$ versus $7.4 \pm 2.4$, respectively $(P<$ $.001)$ at the supraclavicular level. Similarly, in the post-gadoliniumenhanced FS T1-weighted sequence (SPIR Gad-T1WI versus mDixon Gad-T1WI TSE), the ratios measured $0.9 \pm 0.7$ versus $3.7 \pm$ 1.4 , respectively $(P<.001)$ at submandibular level and $0.5 \pm 0.3$ versus $4.3 \pm 2.0$, respectively $(P<.001)$ at supraclavicular level. 


\begin{tabular}{|c|c|c|c|}
\hline \multirow[b]{2}{*}{ Variable } & \multicolumn{2}{|c|}{ Sequence Group } & \multirow[b]{2}{*}{$P$ Value } \\
\hline & $\begin{array}{c}\text { Group B } \\
(n=31)\end{array}$ & $\begin{array}{c}\text { Group A } \\
(n=33)\end{array}$ & \\
\hline \multicolumn{4}{|l|}{ Sex } \\
\hline Male & $20(64.5)$ & $23(69.7)$ & .79 \\
\hline Female & $11(35.5)$ & $10(30.3)$ & \\
\hline Age $(y r)$ & $61 \pm 15$ & $55 \pm 17$ & .15 \\
\hline \multicolumn{4}{|l|}{ Body habitus } \\
\hline AP neck diameter at the level of mandible (C2-3) (mm) & $185 \pm 21$ & $188 \pm 21$ & .28 \\
\hline AP diameter of midneck (C4-5) (mm) & $127 \pm 22$ & $124 \pm 19$ & .90 \\
\hline Shoulder width (mm) & $394 \pm 35$ & $394 \pm 45$ & .84 \\
\hline AP neck diameter at the level of mandible-to-shoulder width ratio & $1.48 \pm 0.15$ & $1.53 \pm 0.16$ & .35 \\
\hline Shoulder width-to midneck AP diameter ratio & $3.16 \pm 0.40$ & $3.21 \pm 0.36$ & .88 \\
\hline
\end{tabular}

a Values are No. (\%) or mean \pm SD unless otherwise specified.

${ }^{\mathrm{b}}$ Mann-Whitney $U$ test.

Table 3: Objective signal intensity ratios ${ }^{\mathrm{a}}$

\begin{tabular}{llll}
\hline \multicolumn{1}{c}{ Variable } & \multicolumn{2}{c}{ Sequence Group } & \\
\cline { 2 - 3 } & $\begin{array}{c}\text { Group B } \\
(\boldsymbol{n}=31)\end{array}$ & $\begin{array}{c}\text { Group A } \\
(\boldsymbol{n}=33)\end{array}$ & P Value \\
\hline T2WI spinal cord-to-fat ratio & $5.7 \pm 1.6$ & $3.5 \pm 3.4$ & $<.001$ \\
$\quad$ Submandibular level & $7.4 \pm 2.4$ & $3.3 \pm 3.4$ & $<.001$ \\
$\quad$ Supraclavicular level & $3.7 \pm 1.4$ & $0.9 \pm 0.7$ & $<.001$ \\
$\begin{array}{l}\text { Post-Gad-TIWI spinal cord-to-fat ratio } \\
\text { Submandibular level }\end{array}$ & $4.3 \pm 2.0$ & $0.5 \pm 0.3$ & $<.001$ \\
$\quad$ Supraclavicular level & &
\end{tabular}

a Values are mean \pm SD unless otherwise specified.

${ }^{\mathrm{b}}$ Mann-Whitney U test.

Table 4: Subjective assessment of image quality, fat suppression, and susceptibility artifacts $^{\mathrm{a}}$

\begin{tabular}{|c|c|c|c|}
\hline \multirow[b]{2}{*}{ Variable } & \multicolumn{2}{|c|}{ Sequence Group } & \multirow[b]{2}{*}{$P$ Value } \\
\hline & $\begin{array}{c}\text { Group B } \\
(n=31)\end{array}$ & $\begin{array}{c}\text { Group A } \\
(n=33)\end{array}$ & \\
\hline \multicolumn{4}{|l|}{ T2WI/STIR images } \\
\hline Overall image-quality grade & $3.9 \pm 0.5$ & $3.6 \pm 0.7$ & .022 \\
\hline \multicolumn{4}{|l|}{ Fat-saturation grade } \\
\hline Maxillary region & $4.6 \pm 0.4$ & $4.3 \pm 0.5$ & .013 \\
\hline Mandibular region & $4.4 \pm 0.5$ & $4.0 \pm 0.6$ & .007 \\
\hline Lower neck region & $4.7 \pm 0.4$ & $4.3 \pm 0.4$ & .001 \\
\hline Dental amalgam susceptibility artifacts (\%) & $38.7 \%$ & $22.7 \%$ & .056 \\
\hline \multicolumn{4}{|l|}{ Post-Gad-TiWls } \\
\hline Overall image-quality grade & $4.0 \pm 0.4$ & $2.6 \pm 0.6$ & $<.001$ \\
\hline \multicolumn{4}{|l|}{ Fat-saturation grade } \\
\hline Maxillary region & $4.8 \pm 0.3$ & $3.8 \pm 0.7$ & $<.001$ \\
\hline Mandibular region & $4.7 \pm 0.3$ & $2.8 \pm 0.5$ & $<.001$ \\
\hline Lower neck region & $4.8 \pm 0.3$ & $1.4 \pm 0.7$ & $<.001$ \\
\hline Dental amalgam susceptibility artifacts (\%) & $37.1 \%$ & $31.8 \%$ & .50 \\
\hline
\end{tabular}

\section{Subjective Assessment}

The averages of the 2 readers' subjective assessments of the uniformity of fat suppression and overall image quality for groups $\mathrm{A}$ and $\mathrm{B}$ are summarized in Table 4.

Fat Suppression. The average scores from both the readers for fat suppression at all 3 levels (maxillary, mandibular, and lower neck region) were significantly higher for mDixon T2-weighted FS TSE (mean, 4.4-4.7) compared with STIR (mean, 4.0-4.3; $P<.013$ for all regions) (Fig 3 ) and mDixon Gad-T1WI FS TSE sequences (mean, 4.7-4.8) compared with SPIR Gad-T1WI (mean, 1.4-3.8, $P<.001$ for all regions) (Figs 4 and 5).
Overall Image Quality. Similarly, the 2 readers' average scores for overall image quality were significantly higher for mDixon T2-weighted FS TSE than for STIR (mean, 3.9 versus 3.6; $P=.022$ ) and mDixon Gad-T1WI FS TSE sequences (mean, 4.0 versus $2.6 ; P<$ $.001)$.

Susceptibility Artifacts Related to Dental Amalgam. There was no significant difference in the percentage of subjects with susceptibility artifacts related to dental amalgam between the 2 groups for both fluid-sensitive sequences $(38.7 \%$ versus $22.7 \%, P=$ $.056)$ and post-Gad-T1WI sequences (37.1\% versus $31.8 \%, P=.5$ ).

\section{Interreader Agreement}

The percentage agreement for the uniformity of fat suppression between the readers was $>87 \%$ for group B patients (On-line Table), though the corresponding $\kappa$ values ranged from 0.05 to 0.15 . Across all 3 stations, readers gave ratings of only $4-5$ for $87 \%-100 \%$ of cases, so there was a limited range of ratings for the $\kappa$ assessment. Group A interreader agreement varied between 25\% and 91\% (On-line Table), with corresponding $\kappa$ values from -0.07 to 0.58 . Across the 3 stations, readers used only 2 different levels $67 \%-$ $99 \%$ of the time.

Scan Acquisition Times. The acquisition times were shorter for the mDixon techniques compared with STIR (2 minutes versus 4 minutes 56 seconds) and SPIR (2 minutes versus 3 minutes).

\section{DISCUSSION}

In this retrospective study, we enrolled 33 subjects who underwent MR imaging with a STIR and SPIR combination of fat-suppression techniques and 31 subjects with mDixon as the fat-sup- 

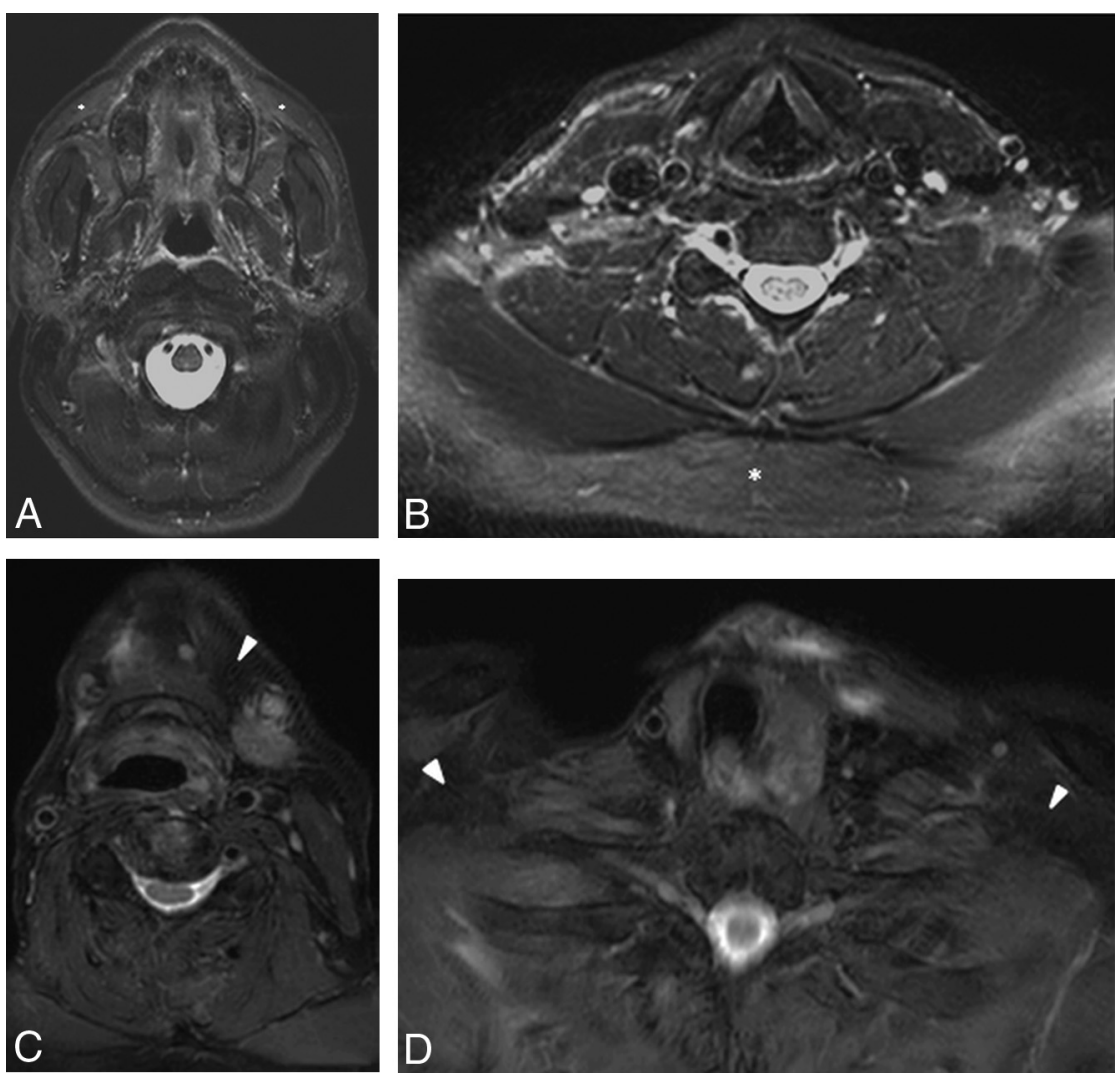

FIG 3. Axial STIR $(A$ and $B)$ and mDixon T2-weighted $(C$ and $D)$ MR images. Note incomplete fat suppression (asterisks) in the maxillary and supraclavicular regions on the STIR technique and complete uniform fat suppression (arrowheads) in the submandibular and supraclavicular regions on the mDixon technique.

pression technique. The subjects were matched for age, sex, and surrogate imaging markers of body habitus in the area of interest. In this study group, we demonstrate that the objective image quality measured for signal intensity ratios (spinal cord to subcutaneous fat signal) was significantly higher for the Dixon technique compared with STIR and SPIR. This finding clearly indicates that the mDixon technique provides better fat suppression, even in the areas where other fat-suppression techniques failed due to technical reasons. In the subjective assessment, readers scored the mDixon technique significantly higher for uniformity of fat suppression and overall image quality. An additional minor advantage with the mDixon technique is relatively shorter scan acquisition times. Our study results are in concordance with previous studies comparing the 3-point mDixon with the CHESS fat-suppression technique in spine, neck, and orbit imaging. ${ }^{3-5}$

The main disadvantages associated with STIR include suppression of signals from tissues with similar T1 values (such as subacute hematoma and gadolinium-enhanced tissues). In addition, fewer sections were obtained for a given TR compared with the spin-echo technique because a certain portion of the time is consumed by the TI and TE of STIR. STIR is considered sensitive to spatial nonuniformity of the applied radiofrequency pulse (unless an adiabatic pulse is used). If the strength of the radiofrequency pulse varies from one position to another within the subject, then the tip angle of the inversion pulse, and hence the quality of fat suppression, will also vary with position. Finally, the uniformity of fat suppression may depend on selection of an appropriate
TI. $^{6-9}$ In addition, STIR alters signal from all tissues and thus decreases the contrast as well as the signal-to-noise ratio. ${ }^{2}$ CHESS and its derivative SPIR hybrid techniques require a homogeneous magnetic field for uniform fat suppression. They fail to suppress fat signal around susceptibility distortions due to metallic hardware, sinuses, and skull base or in the regions far from the isocenter. They also increase the specific absorption rate to the patient and scan times due to use of an extra presaturation pulse and dephasing gradient. Uniformity of FS by CHESS/SPIR techniques is heavily dependent on homogeneity of the main magnetic field (B0) and radiofrequency magnetic field (B1); hence, nonuniform fat suppression occurs farther away from the isocenter of B0. Another important factor described to explain the nonuniformity of FS in the CHESS/SPIR technique in areas with a sharp variation of the shape of anatomic structures such as the floor of the mouth and the supraclavicular region is the so-called bulk susceptibility phenomenon. $^{6}$

The mDixon technique for FS was first described by Dixon in $1984 .{ }^{10}$ This is a spectroscopic imaging technique that relies on water and fat chemical shift differences. The original technique was designed to acquire 2 sets of images, one with water and fat signal being inphase and the other acquired when water and fat signals are at $180^{\circ}$ out-of-phase (referred to as the "2-point Dixon technique"). Using these 2 sets of images, one can generate water-only and fat-only images. ${ }^{11}$ The water-only images serve as effective fat suppression. ${ }^{3,4,12}$ The main advantage of this technique is that it is relatively insensitive to $\mathrm{B} 0$ inhomogeneity but not completely immune to it because sometimes the B0 inhomogeneity can manifest as phase errors. The fundamental assumption of the mDixon technique is that water and fat are the only 2 signal-contributing chemical species in the object to be imaged. Under this assumption, it is believed that water or fat each has only a single spectral peak. This assumption may be true for water but not for fat because fat is known to contain many spectral components. The B0 inhomogeneity and other system imperfections contribute to phase error results in signal contributions to both water-only and fat-only images, even from the pixels containing only fat tissue. ${ }^{2}$

Failure of phase correction usually leads to swapping of water and fat assignments for the affected pixels, which can sometimes present a "pseudomass" appearance or incomplete fat suppression. Correlating with both water-only and fat-only images may help reduce this misinterpretation. A more recent technical advance, the 3-point Dixon technique, acquires an addi- 

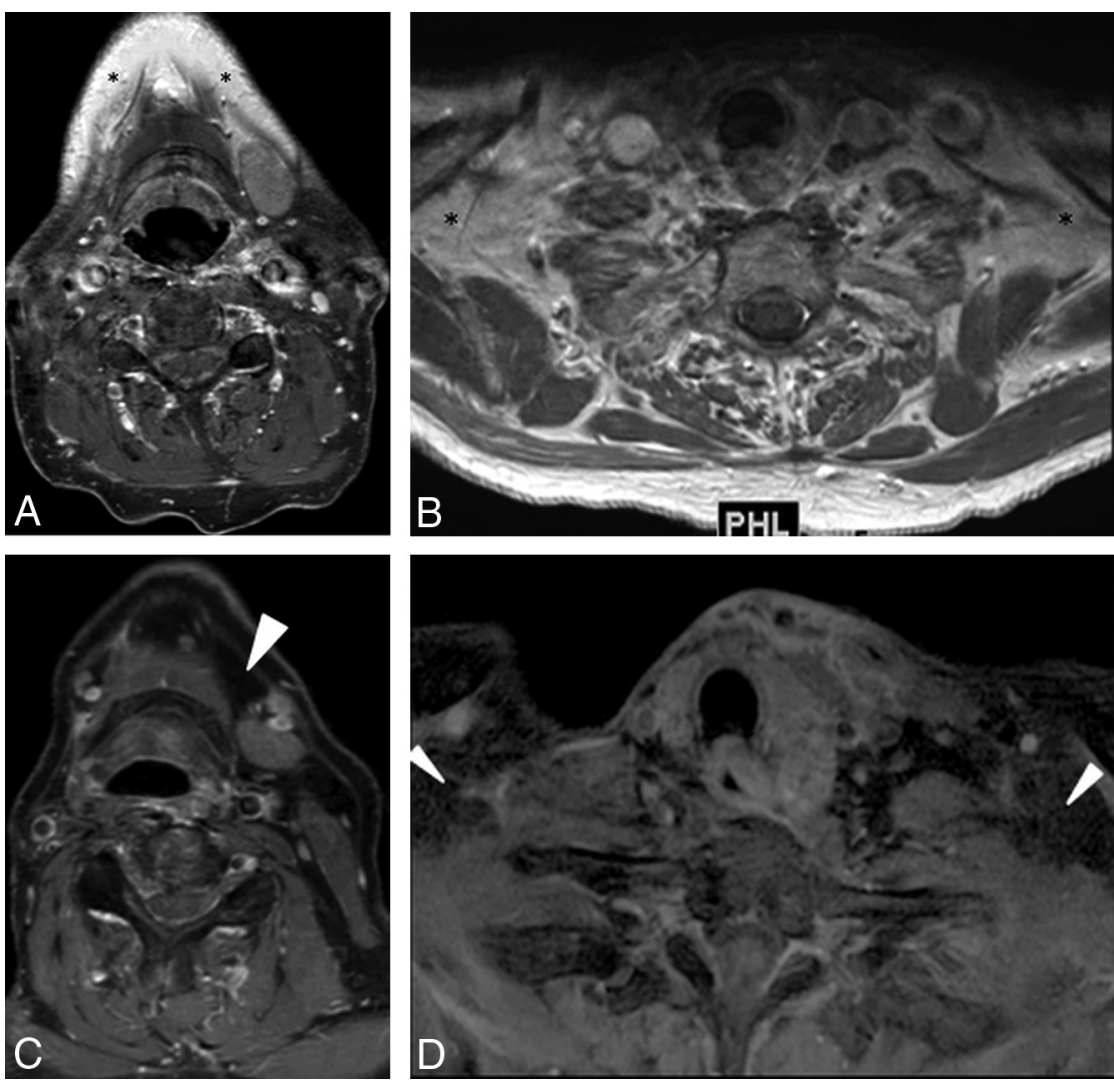

FIG 4. Gadolinium-enhanced axial TI-weighted MR images with SPIR ( $A$ and $B)$ and $m D i x o n(C$ and $D$ ) techniques for fat suppression. Note incomplete fat suppression (asterisks) in the submandibular and supraclavicular regions on the SPIR technique and complete uniform fat suppression (arrowheads) in similar regions on the mDixon technique.
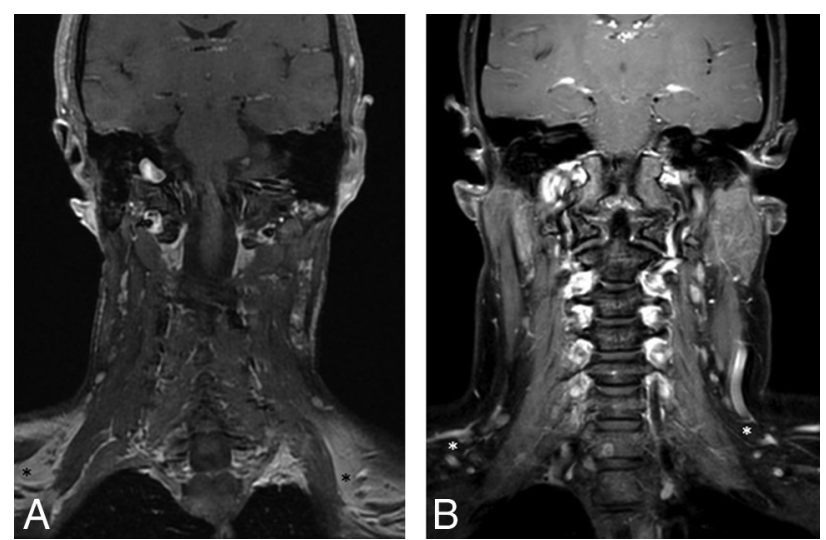

FIG 5. Gadolinium-enhanced coronal T1-weighted MR images with SPIR $(A)$ and mDixon $(B)$ techniques for fat suppression. Note incomplete fat suppression (dark asterisk) in the supraclavicular regions on the SPIR technique and complete uniform fat suppression (white asterisk) in similar regions on the mDixon technique.

tional third set of images along with the traditionally acquired $0^{\circ}$ and $180^{\circ}$; it can be either $-180^{\circ}, 0,180^{\circ}$ or $0,180^{\circ}, 360^{\circ}$. This additional image set helps determine and correct the phase error. $^{13-17}$ The 3-point Dixon technique is considered less prone to phase error, though it is not completely immune to it. This technique can be applied for both $2 \mathrm{D}$ and 3D imaging with different types of pulse sequences, including spin-echo and gradient-based echo sequences. ${ }^{2}$

\section{Study Limitations}

There are several limitations to this study: 1) It is a retrospective study and hence has a limitation of selection bias; 2) it is a relatively small cohort of patients, particularly when considering comparison for 3 different techniques; and 3) a combination of different FS techniques was used in 2 separate populations (groups A and B). This is particularly important because uniformity of FS in certain techniques such as CHESS and its modifications (SPIR) depend heavily on the patient's body habitus and on patient position in the magnet. In our study, we think the contribution of patient-related factors was not significant, considering that there was no statistically significant difference in the demographics and patient body habitus (in the area of interest) between the groups. Fourth, lesion detectability and conspicuity were not assessed due to heterogeneity in the scan indications. Not all patients had a focal lesion, and when a focal lesion was present, no 2 lesions were comparable due to heterogeneity in the type of disease, location, and stage of treatment. Fifth, there was some disagreement between readers during the subjective assessment; however, ratings by both readers showed similar trends between groups $A$ and $\mathrm{B}$, and the readers typically used only 2 different adjacent rating levels at each station. Therefore, while readers may have disagreed on individual ratings, they usually agreed that ratings were high (4-5), low (1-2), or moderate (2-3 or 3-4).

\section{CONCLUSIONS}

The mDixon technique provides more uniform fat suppression and improved image quality compared with other commonly used FS techniques such as STIR and SPIR, while reducing sequence acquisition times in head and neck MR imaging.

Disclosures: Daniel S. Hippe—UNRELATED: Grants/Grants Pending: GE Healthcare, Philips Healthcare, Toshiba America Medical Systems, Comments: for statistical work on other studies.

\section{REFERENCES}

1. Del Grande FD, Santini F, Herzka DA, et al. Fat-suppression techniques for 3-T MR imaging of the musculoskeletal system. Radiographics 2014;34:217-33 CrossRef Medline

2. Ma J. Dixon techniques for water and fat imaging. J Magn Reson Imaging 2008;28:543-58 CrossRef Medline

3. Ma J, Singh SK, Kumar AJ, et al. T2-weighted spine imaging with a fast three-point Dixon technique: comparison with chemical shift selective fat suppression. J Magn Reson Imaging 2004;20:1025-29 CrossRef Medline

4. Ma J, Jackson EF, Kumar AJ, et al. Improving fat-suppressed T2weighted imaging of the head and neck with 2 fast spin-echo Dixon

AJNR Am J Neuroradiol 39:362-68 Feb 2018 www.ajnr.org 
techniques: initial experiences. AJNR Am J Neuroradiol 2009;30: 42-45 Medline

5. Rybicki FJ, Mulkern RV, Robertson RL, et al. Fast three-point Dixon MR imaging of the retrobulbar space with low-resolution images for phase correction: comparison with fast spin-echo inversion recovery imaging. AJNR Am J Neuroradiol 2001;22:1798-802

6. Tien RD. Fat-suppression MR imaging in neuroradiology: techniques and clinical application. AJR Am J Roentgenol 1992;158: 369-79 CrossRef Medline

7. Bydder GM, Steiner RE, Blumgart LH, et al. MR imaging of the liver using short TI inversion recovery sequences. J Comp Assist Tomogr 1985;9:1084-89 CrossRef Medline

8. Bydder GM, Young IR. MR imaging: clinical use of the inversion recovery sequence. J Comp Assist Tomogr 1985;9:659-75 CrossRef Medline

9. Shuman WP, Baron RL, Peters MJ, et al. Comparison of STIR and spin-echo MR imaging at $1.5 \mathrm{~T}$ in 90 lesions of the chest, liver, and pelvis. AJR Am J Roentgenol 1989;152:853-59 CrossRef Medline

10. Dixon WT. Simple proton spectroscopic imaging. Radiology 1984; 153:189-94 CrossRef Medline

11. Hardy PA, Hinks RS, Tkach JA. Separation of fat and water in fast spin-echo MR imaging with the three-point Dixon technique. $J$ Magn Reson Imaging 1995;5:181-85 CrossRef Medline
12. Low RN, Austin MJ, Ma J. Fast spin-echo triple echo Dixon: initial clinical experience with a novel pulse sequence for simultaneous fat-suppressed and nonfat-suppressed T2-weighted spine magnetic resonance imaging. J Magn Reson Imaging 2011;33:390-400 CrossRef Medline

13. Yeung HN, Kormos DW. Separation of true fat and water images by correcting magnetic field inhomogeneity in situ. Radiology 1986; 159:783-86 CrossRef Medline

14. Glover GH, Schneider E. Three-point Dixon technique for true water/fat decomposition with B0 inhomogeneity correction. Magn Reson Med 1991;18:371-83 CrossRef Medline

15. Glover GH. Multipoint Dixon technique for water and fat proton and susceptibility imaging. J Magn Reson Imaging 1991;1:521-30 CrossRef Medline

16. Maas M, Hollak CE, Akkerman EM, et al. Quantification of skeletal involvement in adults with type I Gaucher's disease: fat fraction measured by Dixon quantitative chemical shift imaging as a valid parameter. AJR Am J Roentgenol 2002;179:961-65 CrossRef Medline

17. Wang Y, Li D, Haacke EM, et al. A three-point Dixon method for water and fat separation using $2 \mathrm{D}$ and $3 \mathrm{D}$ gradient-echo techniques. J Magn Reson Imaging 1998;8:703-10 CrossRef Medline 